Published in final edited form as:

J Neurooncol. 2015 January ; 121(1): 159-165. doi:10.1007/s11060-014-1617-9.

\title{
Ipilimumab and whole brain radiation therapy for melanoma brain metastases
}

\author{
Naamit K. Gerber, \\ Department of Radiation Oncology, Memorial Sloan-Kettering Cancer Center, 1275 York Ave, \\ New York, NY 10065, USA
}

\section{Robert J. Young,}

Department of Radiology, Memorial Sloan-Kettering Cancer Center, New York, NY 10065, USA

\section{Christopher A. Barker,}

Department of Radiation Oncology, Memorial Sloan-Kettering Cancer Center, 1275 York Ave, New York, NY 10065, USA

\section{Jedd D. Wolchok,}

Department of Medicine and Ludwig Center, Memorial Sloan-Kettering Cancer Center, New York, NY 10065, USA. Weill Cornell Medical College, New York, NY 10065, USA

\section{Timothy A. Chan,}

Department of Radiation Oncology, Memorial Sloan-Kettering Cancer Center, 1275 York Ave, New York, NY 10065, USA

\section{Yoshiya Yamada,}

Department of Radiation Oncology, Memorial Sloan-Kettering Cancer Center, 1275 York Ave, New York, NY 10065, USA

\section{Leigh Friguglietti, and}

Department of Radiation Oncology, Memorial Sloan-Kettering Cancer Center, 1275 York Ave, New York, NY 10065, USA

\section{Kathryn Beal}

Department of Radiation Oncology, Memorial Sloan-Kettering Cancer Center, 1275 York Ave, New York, NY 10065, USA

Kathryn Beal: bealk@mskcc.org

\section{Abstract}

Brain metastases (BM) frequently develop in patients with melanoma and are associated with a poor prognosis. Whole brain radiation therapy (WBRT) is a standard intervention for intracranial disease, particularly in patients with multiple BM. Ipilimumab improves survival in patients with advanced melanoma. The purpose of this study is to investigate the safety and efficacy of concurrent WBRT and ipilimumab. A retrospective analysis was conducted of 13 consecutive patients treated with WBRT within 30 days of ipilimumab administration. Radiographic response,

Correspondence to: Kathryn Beal, bealk@mskcc.org.

Conflict of interest Dr. Wolchok is a paid consultant for and receives research funding from Bristol-Myers Squibb. 
as measured by serial magnetic resonance imaging scans post-treatment, was graded by modified World Health Organization (mWHO) and immune-related response criteria (irRC) in the 9 patients with follow-up imaging. Treatment-related toxicity was prospectively assessed during treatment. Four of nine patients (44\%) experienced partial response or stable central nervous system (CNS) disease as measured by mWHO criteria. This number increased to 5 patients $(56 \%)$ when irRC criteria were used. Rates of treatment-related neurologic toxicity were low with only one patient experiencing grade 3-4 neurologic toxicity. There was a high rate of intratumoral hemorrhage in this patient population, with 10 of 10 patients with post-treatment imaging demonstrating new or increased intratumoral bleeding after WBRT. This retrospective study demonstrates that the primary pattern of CNS response to WBRT and ipilimumab is stable disease and not regression of BM. Furthermore, while the combination of WBRT and ipilimumab may offer promising efficacy, prospective studies are needed to further assess efficacy and toxicity.

\section{Keywords}

Melanoma; Brain metastases; Ipilimumab; Immunotherapy; Whole brain radiotherapy

\section{Introduction}

Brain metastases $(\mathrm{BM})$ are common in patients with melanoma with incidence varying from 10 to $40 \%$ [1]. Prognosis is poor with a median survival of 4-5 months [2, 3]. Large retrospective series have demonstrated improved outcomes with whole-brain radiotherapy (WBRT) $[2,4]$ and a prospective randomized trial demonstrated longer time to intracranial progression in patients who received WBRT and chemotherapy compared with chemotherapy alone [5]. Yet, studies of WBRT in patients with metastatic melanoma have shown median survival of 3.5-4 months [6-9] with local control rates ranging from 0 to $61 \%[8,10,11]$. Though there is demonstrated benefit for neurosurgical resection and stereotactic radiosurgery (SRS) in patients with a limited number of BM, the options for patients with multiple BM or a performance status that precludes surgical or radiosurgical intervention remain limited $[6,12,13]$. A more recent development is the use of BRAF inhibitors in patients with BRAF V600E mutated melanoma with BM, an area of ongoing research [14].

With the recent approval of ipilimumab to treat advanced melanoma based on Phase III [15, 16] trials and preclinical evidence that activated $T$ cells can cross the blood-brain barrier $[17,18]$, there is interest in using ipilimumab to treat BM from metastatic melanoma. Though there is some evidence that fractionated radiation may negatively affect the immune system [19], there is also evidence for a positive biologic interaction between radiation and immunotherapy [20]. Retrospective data demonstrated safety and clinical activity of ipilimumab in melanoma patients with BM [21]. A recent Phase II trial investigated ipilimumab in patients with melanoma and BM and found a disease control rate of $24 \%$ in the brain in neurologically asymptomatic patients not receiving corticosteroid treatment [22]. There was no unexpected toxicity. 
The data on the combination of radiation and ipilimumab in patients with BM is limited [20]. Retrospective reports on the combination of ipilimumab and SRS have shown conflicting results, with some demonstrating an association between combined ipilimumab and SRS with prolonged survival, even when controlling for graded prognostic assessment (GPA) [23, 24], and others showing no difference in survival between SRS alone and SRS in combination with ipilimumab [25]. Given the higher fraction size utilized in SRS as compared to WBRT, the biologic basis for the interaction of radiation and ipilimumab may differ between SRS and WBRT. Other than individual case reports [26, 27], there have been no data reported on the combination of WBRT and ipilimumab.

Given this lack of data and the promising results from the Phase II study of ipilimumab alone for BM, we examined our experience with the combination of WBRT and ipilimumab. We aimed to assess clinical response, radiographic response, and toxicity.

\section{Methods}

After obtaining institutional review board approval and a waiver of informed consent, we identified all patients with metastatic melanoma and BM diagnosed during 2002-2011 treated with both ipilimumab and WBRT from an institutional database. Twenty-nine patients were identified, of whom 13 received ipilimumab and WBRT within 30 days of one another. Thirty days was chosen as a cutoff because of a half-life of ipilimumab of about 15 days [28].

Of these 13 patients, the majority $(n=8)$ never received prior radiation to the brain. Five patients underwent a craniotomy and tumor resection prior to WBRT, and 5 were previously treated with SRS. The median dose of WBRT was 3,000 cGy (range, 2,700-3,750 cGy) delivered in 9-15 (median 10) fractions. Twelve patients were treated with $3 \mathrm{mg} / \mathrm{kg}$ of ipilimumab with one receiving $10 \mathrm{mg} / \mathrm{kg}$. Patients were treated with $2(\mathrm{n}=4)$ or $4(\mathrm{n}=9)$ doses of ipilimumab. The 4 patients who received 2 doses all had extracranial disease progression which is why they did not receive further treatment with ipilimumab. Five patients received WBRT concurrent with ipilimumab $(n=5)$ or between cycles of ipilimumab $(n=1)$. Four patients received ipilimumab prior to WBRT with a mean of 13.5 days between last ipilimumab dose and the start of WBRT. Three patients received WBRT prior to ipilimumab with a mean of 14 days between the end of WBRT and the first dose of ipilimumab.

Patients were characterized according to melanoma-specific GPA [29]. All patients had brain imaging prior to WBRT. Ten patients had brain imaging after WBRT, 9 with magnetic resonance imaging (MRI) and 1 with computed tomograph (CT). A total of 40 post-WBRT MRIs were available in 9 patients with a median of 3 scans per patient. For this study, all scans were assessed by a neuroradiologist (RY) and radiographic response was determined by both $\mathrm{mWHO}$ and immune-related response criteria (irRC). The sum of the products (SPD) of two-dimensional tumor measurements were obtained for each scan. While both systems consider a $\geq 50 \%$ decrease in the SPD of all index lesions a partial response (PR) and at least a $25 \%$ increase in SPD compared with nadir progressive disease (PD), the irRC do not consider all new lesions PD and require two consecutive observations of $25 \%$ growth 
to qualify as PD [30]. If only one scan was available post-treatment and it demonstrated PD, patients were classified as having PD by both $\mathrm{mWHO}$ and irRC. Time to intracranial progression was determined by Kaplan-Meier method with date of last scan used as the last follow-up point.

Extracranial response was assessed with irRC using all available post-treatment imaging. One patient did not have any evidence of extracranial disease and was treated with ipilimumab for central nervous system (CNS) disease alone. Of the remaining 12 patients, 10 had post-treatment imaging. Patients were assessed for best overall response rate.

Intratumoral hemorrhage was determined by a neuroradiologist (RY) after inspection of susceptibility weighted $(n=8)$, or $b-0$ diffusion-weighted $(n=1)$ images and confirmed by inspection of the T1-weighted and T2-weighted images $(n=9)$. One patient had no MRI post-treatment but had a CT scan showing increased hyperdense blood products consistent with increased hemorrhage post-treatment. Patients were coded as having new hemorrhage if a lesion did not have any baseline hemorrhage or as having increased hemorrhage if a hemorrhagic lesion at baseline had increased signal changes. Hemorrhage has variable appearances due to the timing and stage of the blood products, as well as the applied pulse sequence, but subacute hemorrhage often manifests as hypointense signal on susceptibility weighted and diffusion-weighted images and hyperintense signal on T1-weighted images. Overlapping imaging characteristics have been described in melanotic metastases that contain $>10 \%$ melanin-containing cells due to intrinsic $\mathrm{T} 1$ and $\mathrm{T} 2$ shortening effects from paramagnetic melanin pigments $[31,32]$. It is therefore possible that some of the metastases coded as hemorrhagic before treatment also contained melanin-rich cells, as there are no reliable imaging techniques to distinguish between the two. Since our intent was to describe the change and possible relationship between hemorrhage and treatment, we recorded new or increased signal changes during treatment as new or increased hemorrhage, respectively, with the assumption that the number of melanin-rich cells will not increase with treatment.

All patients had weekly, standardized prospective assessment of neurologic symptoms during WBRT except 2 patients who were hospitalized and thus had daily progress note documentation during radiation. Patients were followed after WBRT by their radiation, medical, and neuro-oncologists and all toxicity data in the 3 months following WBRT or 70 days after last ipilimumab dose was recorded as treatment-related toxicity. Corticosteroid dosing at time of WBRT and in the 6-8 weeks following radiation was obtained. Adverse events were graded according to the National Cancer Institute's Common Toxicity Criteria for Adverse Events (CTCAE) version 4.0. Due to varying length and frequency of follow-up among patients immediately following WBRT, we do not report late toxicity.

\section{Results}

Patient characteristics are summarized in Table 1. The median age was 64 (range, 36-85) years with a roughly even distribution of men and women. The majority of patients were GPA class 1-2 (69\%) and patients had a median of 7 BM (range, 1-31 BM). All but five patients had been treated with prior systemic therapy. Median follow-up was 4 months for all patients and 30 months in the one patient alive at time of analysis. 
Ten of 13 patients (77\%) received dexamethasone during WBRT. Dose and reason for steroid use was known for 9 of these 10 patients. The median dose was $8 \mathrm{mg}$ daily. Five patients received dexamethasone for palliation and four to prevent brain edema. Five patients (50\%) were off corticosteroids completely by $6-8$ weeks post-WBRT, $2(20 \%)$ were on lower doses by $6-8$ weeks post-WBRT, and corticosteroid information post-WBRT was not available in $3(30 \%)$. Six patients were asymptomatic at baseline. Two patients had initial worsening of baseline neurologic symptoms during WBRT with gradual improvement in the weeks following WBRT. One patient had initial improvement 6-8 weeks post-WBRT but worsening at 4 months post-treatment. One patient had no improvement of baseline symptoms. Two other patients were dead of disease shortly after WBRT.

Radiographic response was determined by both $\mathrm{mWHO}$ and irRC criteria on the first scan following WBRT (median 7.7; range 4.3-11.7 weeks after WBRT). There was 1 patient with a PR (11\%), 6 patients with SD (66\%), and 2 patients with PD using both criteria (22\%), yielding a rate of CNS disease control of $78 \%$. When overall rates of progression were assessed using all available post-treatment scans, 3 of the patients with SD progressed using $\mathrm{mWHO}$ criteria and 2 progressed using irRC criteria, yielding a CNS disease-control rate of $44 \%$ by mWHO criteria and $56 \%$ by irRC criteria. Median time to intracranial progression was 102 days by $\mathrm{mWHO}$ criteria and 349 days by irRC criteria. Only two patients developed new BM after WBRT.

The change from baseline of the SPD of the BM is graphed in Fig. 1 for the 4 patients with the highest number of scans post-treatment. These four panels represent different patterns of response including SD by irRC (1a and 1b), PD (1c), and PR (1d). Only one patient had a different classification depending on criteria used, PD by mWHO criteria and SD by irRC (Fig. 1a). Though the patient had a $33 \%$ increase in the SPD of her BM (Fig. 2a and 2b), subsequent consecutive scans did not show a $>25 \%$ increase, required for a designation of PD by irRC. This patient was the only patient alive at data analysis, with a follow-up of 30 months. Her clinical course has been consistent with SD with no new neurologic symptoms or further consecutive increase of her BM $>25 \%$. An FDG positron-emission tomography (PET) brain scan was obtained to assess for viable tumor [33] and the lesions were found to be hypometabolic (Fig. 2c-e). Her clinical course and lack of viable tumor on PET scan support the designation of SD by irRC.

Median overall survival was 4 months for the cohort with a 1-year survival rate of $15.4 \%$. Extracranial best overall response rate was measured in the 10 patients with post-ipilimumab extracranial imaging. There was one CR (10\%), one PR (10\%), 2 patients with SD (20\%) and 5 patients with PD (50\%).

Treatment-related toxicity is detailed in Table 2 . Grade 1-2 fatigue, nausea, and cognitive changes occurred in 54, 23, and $23 \%$ of all patients, respectively. Three patients ( $23 \%$ ) experienced grade 1-2 visual changes or diplopia, which were pre-existing in one of these patients. Two patients experienced grade 2 seizure during the course of WBRT $(15 \%)$. One of these patients had recently undergone a craniotomy and had seizures prior to surgery. A second patient had documented hemorrhage during WBRT, likely the cause of the acute seizure. This patient also experienced grade 3 cognitive change during the WBRT. 
All of the patients with follow-up imaging post-WBRT $(n=10)$ were noted to have new or increased intra-tumoral hemorrhage. Of the eight patients with hemorrhagic lesions at baseline, all had increases in intra-tumoral hemorrhage on post-treatment imaging. Two of these also developed hemorrhage in lesions non-hemorrhagic at baseline. Both of the patients with no hemorrhagic lesions at baseline developed hemorrhage on post-treatment imaging. The median time to development of new or worsening hemorrhage was 53 days (range, 4-201 days). No patient required surgical evacuation and none of the patients were on anticoagulation at the time of hemorrhage. While the majority of the patients were asymptomatic, there were 4 patients with new or worsening neurologic symptoms that correlated with new or worsening hemorrhage. In one patient, the symptoms were attributed to increased edema surrounding the hemorrhagic lesions. A second patient experienced visual changes attributed by the treating neuro-oncologist to either migraines or worsening hemorrhagic BM. The final two patients had acute mental status changes, attributed to the intra-tumoral hemorrhage in one of these cases.

\section{Discussion}

This study reports on a cohort of patients treated with WBRT and ipilimumab within 30 days of one another. WBRT is an accepted treatment for patients with metastatic melanoma and BM, though reports of its efficacy and durability vary. Ipilimumab has proven activity in advanced melanoma and has recently been shown to be both safe and efficacious in treating BM [22]. To our knowledge, this is the first case series of combining these two therapies. This report demonstrates that the combination of WBRT and ipilimumab is efficacious. We report a CNS disease control rate of $78 \%$ on the first post-WBRT scan (mWHO and irRC criteria) and $56 \%$ using all available post-treatment scans (irRC criteria). This is more than double the response rate seen in a Phase II prospective trial of ipilimumab alone for the treatment of BM in which a CNS disease control rate of $25 \%$ using irRC was found in patients with small, asymptomatic BM [22]. This compares favorably to the cerebral control rate of $47 \%$ in the WBRT and fotemustine arm of the phase III trial comparing WBRT to WBRT and concurrent fotemustine [5]. In that study, the median time to intracranial progression in the combined chemoradiation arm was 80 days in the intention to treat population which compares favorably to our median time to intracranial progression of 102 days by mWHO criteria and 349 days by irRC criteria [5]. Though retrospective, a strength of this report is the availability of serial post-treatment MRIs in 9 patients with a mean of 4.4 follow-up scans per patient, which were re-reviewed by a neuroradiologist. Furthermore, treatment-related toxicity data was prospectively gathered while patients were on treatment.

Though we only had a small number of patients in this study, the primary radiographic pattern observed among patients with response to WBRT and ipilimumab was one of SD, often with slight interval enlargement of BM after WBRT. We had no patients with a CR, 1 with a PR, and 5 with SD. This pattern of response was best illustrated by the patient for whom we had the longest follow-up. Though her lesions never disappeared after WBRT, and became up to $33 \%$ larger on follow-up imaging, long follow-up (30 months) with extensive clinical and radiographic evaluation including a PET scan have proven her to have SD with no new neurologic symptoms over time. Though she would have been classified as having PD by mWHO criteria, she was classified as having SD by irRC criteria [30]. We believe our 
overall response pattern of SD, particularly as illustrated by this patient, argue for the use of irRC in future studies of radiation and ipilimumab. This patient's case also illustrates the role of PET scan in evaluating post-WBRT enlargement of BM and suggests a potential role for functional imaging in assessing response to WBRT and ipilimumab.

All patients with follow-up imaging in this study had new or worsening intratumoral hemorrhage. The majority of patients were asymptomatic, though two experienced symptoms likely related to worsening hemorrhage. There is a high risk of bleeding in melanoma BM in the absence of any intervention, with an incidence of about $30 \%$ of patients having radiographically detected hemorrhage $[9,34]$. The trials that study WBRT alone for patients with metastatic melanoma do not report on intratumoral hemorrhage after WBRT [7-9]. A retrospective study on SRS and ipilimumab reported a hemorrhage rate of $42 \%$ in treated BM [24]. A second report on SRS and ipilimumab reported no increase in hemorrhage when ipilimumab was added to SRS [25]. In the phase II study of ipilimumab alone for melanoma BM, one patient was noted to have grade 4 brain hemorrhage attributed to the disease, though ipilimumab could not be ruled out as an etiologic factor [22]. Whether the high rate of hemorrhage observed in our study is due to the natural course of melanoma $\mathrm{BM}$ or whether it is due to the WBRT and/or ipilimumab cannot be determined in this single-arm retrospective study. However, the rate we observed, coupled with previously published data on radiation and ipilimumab, emphasizes the need for prospective study of the combination of cranial irradiation and ipilimumab with careful assessment of intratumoral hemorrhage. Ongoing trials such as a phase I study of ipilimumab and WBRT or SRS in patients with melanoma and BM (clinicaltrials.gov: NCT01703507) will help clarify the safety of combining ipilimumab and brain radiation.

A limitation of this study is the small size of the described patient cohort and its retrospective nature. Additional limitations include our inability to capture late toxicity and the variability of follow-up in patients in the 3 months post-treatment which limits our assessment of treatment-related toxicity.

In conclusion, this study demonstrates that there may be a role for combining WBRT and ipilimumab in the treatment of patients with metastatic melanoma and multiple BM. The pattern of response seems to largely be one of SD. Future studies are needed to better assess efficacy and toxicity of combining WBRT and ipilimumab with particular attention to rates of intratumoral hemorrhage and careful assessment of radiographic response such that cases of stable disease are not erroneously misclassified as progression.

\section{Acknowledgments}

The authors would like to thank Lawrence A. Herman for his editorial assistance.

\section{References}

1. Bafaloukos D, Gogas H. The treatment of brain metastases in melanoma patients. Cancer Treat Rev. 2004; 30:515-520. [PubMed: 15325032]

2. Fife KM, Colman MH, Stevens GN, Firth IC, Moon D, Shannon KF, Harman R, Petersen-Schaefer K, Zacest AC, Besser M, Milton GW, McCarthy WH, Thompson JF. Determinants of outcome in 
melanoma patients with cerebral metastases. J Clin Oncol. 2004; 22:1293-1300. [PubMed: 15051777]

3. Staudt M, Lasithiotakis K, Leiter U, Meier F, Eigentler T, Bamberg M, Tatagiba M, Brossart P, Garbe C. Determinants of survival in patients with brain metastases from cutaneous melanoma. Br J Cancer. 2010; 102:1213-1218. [PubMed: 20372154]

4. Sampson JH, Carter JH Jr, Friedman AH, Seigler HF. Demographics, prognosis, and therapy in 702 patients with brain metastases from malignant melanoma. J Neurosurg. 1998; 88:11-20. [PubMed: 9420067]

5. Mornex F, Thomas L, Mohr P, Hauschild A, Delaunay MM, Lesimple T, Tilgen W, Bui BN, Guillot B, Ulrich J, Bourdin S, Mousseau M, Cupissol D, Bonneterre ME, De Gislain C, Bensadoun RJ, Clavel M. A prospective randomized multicentre phase III trial of fotemustine plus whole brain irradiation versus fotemustine alone in cerebral metastases of malignant melanoma. Melanoma Res. 2003; 13:97-103. [PubMed: 12569292]

6. Douglas JG, Margolin K. The treatment of brain metastases from malignant melanoma. Semin Oncol. 2002; 29:518-524. [PubMed: 12407517]

7. Ellerhorst J, Strom E, Nardone E, McCutcheon I. Whole brain irradiation for patients with metastatic melanoma: a review of 87 cases. Int J Radiat Oncol Biol Phys. 2001; 49:93-97. [PubMed: 11163501]

8. Hauswald H, Dittmar JO, Habermehl D, Rieken S, Sterzing F, Debus J, Combs SE. Efficacy and toxicity of whole brain radiotherapy in patients with multiple cerebral metastases from malignant melanoma. Radiat Oncol. 2012; 7:130. [PubMed: 22857154]

9. Raizer JJ, Hwu WJ, Panageas KS, Wilton A, Baldwin DE, Bailey E, von Althann C, Lamb LA, Alvarado G, Bilsky MH, Gutin PH. Brain and leptomeningeal metastases from cutaneous melanoma: survival outcomes based on clinical features. Neuro-Oncology. 2008; 10:199-207. [PubMed: 18287337]

10. Meyners T, Heisterkamp C, Kueter JD, Veninga T, Stalpers LJ, Schild SE, Rades D. Prognostic factors for outcomes after whole-brain irradiation of brain metastases from relatively radioresistant tumors: a retrospective analysis. BMC Cancer. 2010; 10:582. [PubMed: 20977700]

11. Rades D, Heisterkamp C, Huttenlocher S, Bohlen G, Dunst J, Haatanen T, Schild SE. Dose escalation of whole-brain radiotherapy for brain metastases from melanoma. Int J Radiat Oncol Biol Phys. 2010; 77:537-541. [PubMed: 19733017]

12. Andrews DW, Scott CB, Sperduto PW, Flanders AE, Gaspar LE, Schell MC, Werner-Wasik M, Demas W, Ryu J, Bahary JP, Souhami L, Rotman M, Mehta MP, Curran WJ Jr. Whole brain radiation therapy with or without stereotactic radiosurgery boost for patients with one to three brain metastases: phase III results of the RTOG 9508 randomised trial. Lancet. 2004; 363:16651672. [PubMed: 15158627]

13. Patchell RA, Tibbs PA, Walsh JW, Dempsey RJ, Maruyama Y, Kryscio RJ, Markesbery WR, Macdonald JS, Young B. A randomized trial of surgery in the treatment of single metastases to the brain. N Engl J Med. 1990; 322:494-500. [PubMed: 2405271]

14. Soffietti R, Trevisan E, Ruda R. Targeted therapy in brain metastasis. Curr Opin Oncol. 2012; 24:679-686. [PubMed: 22820413]

15. Robert C, Thomas L, Bondarenko I, O’Day S, DJM, Garbe C, Lebbe C, Baurain JF, Testori A, Grob JJ, Davidson N, Richards J, Maio M, Hauschild A, Miller WH Jr, Gascon P, Lotem M, Harmankaya K, Ibrahim R, Francis S, Chen TT, Humphrey R, Hoos A, Wolchok JD. Ipilimumab plus dacarbazine for previously untreated metastatic melanoma. N Engl J Med. 2011; 364:25172526. [PubMed: 21639810]

16. Hodi FS, O’Day SJ, McDermott DF, Weber RW, Sosman JA, Haanen JB, Gonzalez R, Robert C, Schadendorf D, Hassel JC, Akerley W, van den Eertwegh AJ, Lutzky J, Lorigan P, Vaubel JM, Linette GP, Hogg D, Ottensmeier CH, Lebbe C, Peschel C, Quirt I, Clark JI, Wolchok JD, Weber JS, Tian J, Yellin MJ, Nichol GM, Hoos A, Urba WJ. Improved survival with ipilimumab in patients with metastatic melanoma. N Engl J Med. 2010; 363:711-723. [PubMed: 20525992]

17. Prins RM, Vo DD, Khan-Farooqi H, Yang MY, Soto H, Economou JS, Liau LM, Ribas A. NK and CD4 cells collaborate to protect against melanoma tumor formation in the brain. J Immunol. 2006; 177:8448-8455. [PubMed: 17142742] 
18. Wilson EH, Weninger W, Hunter CA. Trafficking of immune cells in the central nervous system. J Clin Investig. 2010; 120:1368-1379. [PubMed: 20440079]

19. Grossman SA, Ye X, Lesser G, Sloan A, Carraway H, Desideri S, Piantadosi S. Immunosuppression in patients with high-grade gliomas treated with radiation and temozolomide. Clin Cancer Res. 2011; 17:5473-5480. [PubMed: 21737504]

20. Barker C, Postow M. Combinations of radiotherapy and immunotherapy for melanoma: a review of clinical outcomes. Int J Radiat Oncol Biol Phys. 2014; 88:986-987. [PubMed: 24661650]

21. Weber JS, Amin A, Minor D, Siegel J, Berman D, O’Day SJ. Safety and clinical activity of ipilimumab in melanoma patients with brain metastases: retrospective analysis of data from a phase 2 trial. Melanoma Res. 2011; 21:530-534. [PubMed: 22051508]

22. Margolin K, Ernstoff MS, Hamid O, Lawrence D, McDermott D, Puzanov I, Wolchok JD, Clark JI, Sznol M, Logan TF, Richards J, Michener T, Balogh A, Heller KN, Hodi FS. Ipilimumab in patients with melanoma and brain metastases: an open-label, phase 2 trial. Lancet Oncol. 2012; 13:459-465. [PubMed: 22456429]

23. Knisely JP, Yu JB, Flanigan J, Sznol M, Kluger HM, Chiang VL. Radiosurgery for melanoma brain metastases in the ipilimumab era and the possibility of longer survival. J Neurosurg. 2012; 117:227-233. [PubMed: 22702482]

24. Kiess A, Wolchok J, Barker C, et al. Ipilimumab and stereotactic radiosurgery for melanoma brain metastases. Int J Radiat Oncol Biol Phys. 2012; 84:S115-S116.

25. Mathew M, Tam M, Ott PA, Pavlick AC, Rush SC, Donahue BR, Golfinos JG, Parker EC, Huang PP, Narayana A. Ipilimumab in melanoma with limited brain metastases treated with stereotactic radiosurgery. Melanoma Res. 2013; 23:191-195. [PubMed: 23462208]

26. Muller-Brenne T, Rudolph B, Schmidberger H, et al. Successful therapy of a cerebral metastasized malign melanoma by whole-brain-radiation and therapy with ipilimumab. J Dtsch Dermatol Ges. 2011; 9:787-788.

27. Bot I, Blank CU, Brandsma D. Clinical and radiological response of leptomeningeal melanoma after whole brain radiotherapy and ipilimumab. J Neurol. 2012; 259:1976-1978. [PubMed: 22527228]

28. Weber JS, O’Day S, Urba W, Powderly J, Nichol G, Yellin M, Snively J, Hersh E. Phase I/II study of ipilimumab for patients with metastatic melanoma. J Clin Oncol. 2008; 26:5950-5956. [PubMed: 19018089]

29. Sperduto PW, Kased N, Roberge D, Xu Z, Shanley R, Luo X, Sneed PK, Chao ST, Weil RJ, Suh J, Bhatt A, Jensen AW, Brown PD, Shih HA, Kirkpatrick J, Gaspar LE, Fiveash JB, Chiang V, Knisely JP, Sperduto CM, Lin N, Mehta M. Summary report on the graded prognostic assessment: an accurate and facile diagnosis-specific tool to estimate survival for patients with brain metastases. J Clin Oncol. 2012; 30:419-425. [PubMed: 22203767]

30. Wolchok JD, Hoos A, O’Day S, Weber JS, Hamid O, Lebbe C, Maio M, Binder M, Bohnsack O, Nichol G, Humphrey R, Hodi FS. Guidelines for the evaluation of immune therapy activity in solid tumors: immune-related response criteria. Clin Cancer Res. 2009; 15:7412-7420. [PubMed: 19934295]

31. Isiklar I, Leeds NE, Fuller GN, Kumar AJ. Intracranial metastatic melanoma: correlation between MR imaging characteristics and melanin content. Am J Roentgenol. 1995; 165:1503-1512. [PubMed: 7484597]

32. Premkumar A, Marincola F, Taubenberger J, Chow C, Venzon D, Schwartzentruber D. Metastatic melanoma: correlation of MRI characteristics and histopathology. J Magn Reson Imaging. 1996; 6:190-194. [PubMed: 8851427]

33. Hatzoglou V, Ulaner GA, Zhang Z, Beal K, Holodny AI, Young RJ. Comparison of the effectiveness of MRI perfusion and fluorine-18 FDG PET-CT for differentiating radiation injury from viable brain tumor: a preliminary retrospective analysis with pathologic correlation in all patients. Clin Imaging. 2013; 37:451-457. [PubMed: 23068052]

34. Byrne TN, Cascino TL, Posner JB. Brain metastasis from melanoma. J Neurooncol. 1983; 1:313317. [PubMed: 6678974] 

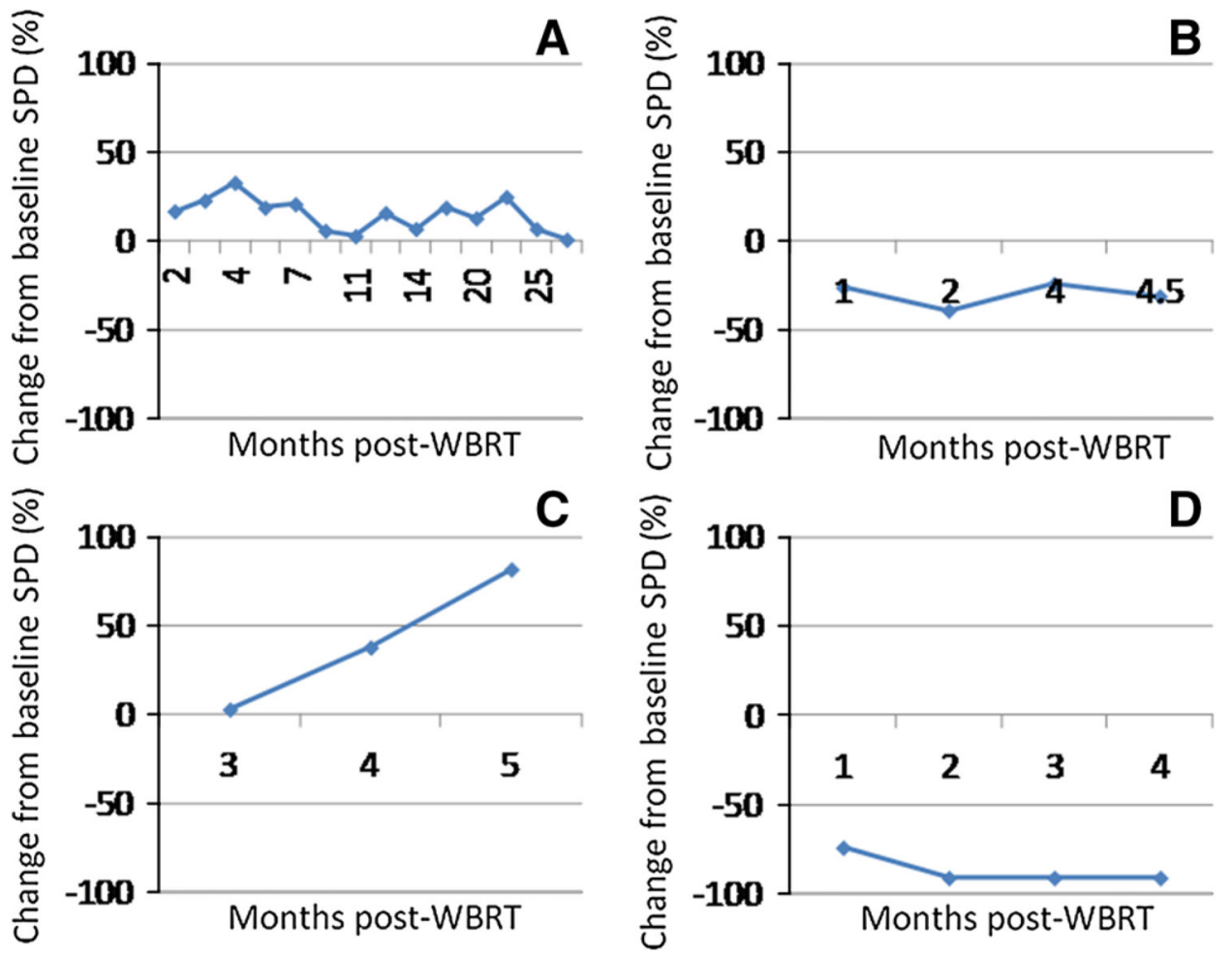

Fig. 1.

Patterns of response to whole-brain radiation therapy and ipilimumab observed in our cohort. Shown are the four patterns observed in our patients. a Stable disease using immunerelated response criteria showing growth at different time points without frank progression. b Stable disease by both immune-related response criteria and modified World Health Organization criteria with overall pattern of shrinkage in the sum of the products (SPD). c Progressive disease. d Partial response. WBRT whole brain radiation therapy 

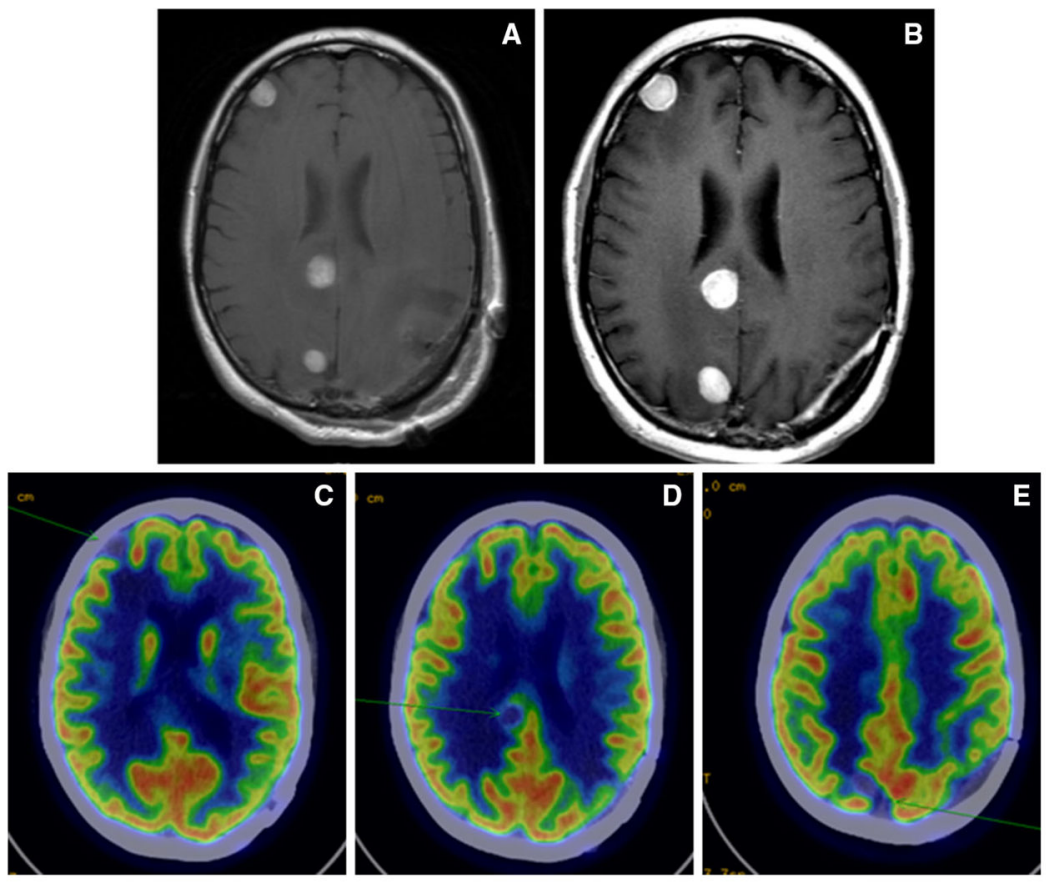

Fig. 2.

Neuroimaging from one patient who was classified as having progressive disease by modified World Health Organization criteria and stable disease by immune-related response criteria. a Contrast T1-weighted image pre-treatment. b Four months post-treatment showing $33 \%$ growth of brain metastases. c-e Positron-emission tomography scan 4-months posttreatment showing that areas of growth on magnetic resonance imaging are photopenic and therefore not likely to represent viable disease 


\section{Table 1}

\section{Patient and treatment characteristics}

\begin{tabular}{|c|c|}
\hline \multicolumn{2}{|l|}{ Age (years) } \\
\hline Median (range) & $64(36-85)$ \\
\hline \multicolumn{2}{|l|}{ Sex } \\
\hline Male & $7(54 \%)$ \\
\hline Female & $6(46 \%)$ \\
\hline \multicolumn{2}{|l|}{ Stage at diagnosis } \\
\hline I-III & $10(77 \%)$ \\
\hline IV & $3(23 \%)$ \\
\hline \multicolumn{2}{|l|}{ GPA class } \\
\hline 1 & $4(31 \%)$ \\
\hline 2 & $5(38 \%)$ \\
\hline 3 & $3(23 \%)$ \\
\hline 4 & $1(8 \%)$ \\
\hline \multicolumn{2}{|l|}{ Number of brain metastases } \\
\hline Median (range) & $7(1-31)$ \\
\hline \multicolumn{2}{|l|}{ Previous systemic therapy } \\
\hline Any & $8(62 \%)$ \\
\hline Targeted agent & $5(38 \%)$ \\
\hline Immunotherapy & $3(23 \%)$ \\
\hline Temozolomide & $7(54 \%)$ \\
\hline Vinblastine & $4(31 \%)$ \\
\hline Cisplatin & $4(31 \%)$ \\
\hline \multicolumn{2}{|l|}{ Previous radiation therapy to brain } \\
\hline None & $8(62 \%)$ \\
\hline Stereotactic radiosurgery & $4(31 \%)$ \\
\hline Hypofractionated radiation therapy & $1(8 \%)$ \\
\hline Previous craniotomy & $5(38 \%)$ \\
\hline \multicolumn{2}{|l|}{ WBRT dose (cGy) } \\
\hline Median (range) & $3,000(2,700-3,750)$ \\
\hline \multicolumn{2}{|l|}{ Ipilimumab dose } \\
\hline $3 \mathrm{mg} / \mathrm{kg}$ & $12(92 \%)$ \\
\hline $10 \mathrm{mg} / \mathrm{kg}$ & $1(8 \%)$ \\
\hline \multicolumn{2}{|l|}{ Ipilimumab cycles } \\
\hline Median (range) & $4(2-4)$ \\
\hline
\end{tabular}

Interval between WBRT and ipilimumab (days) 
Table 2

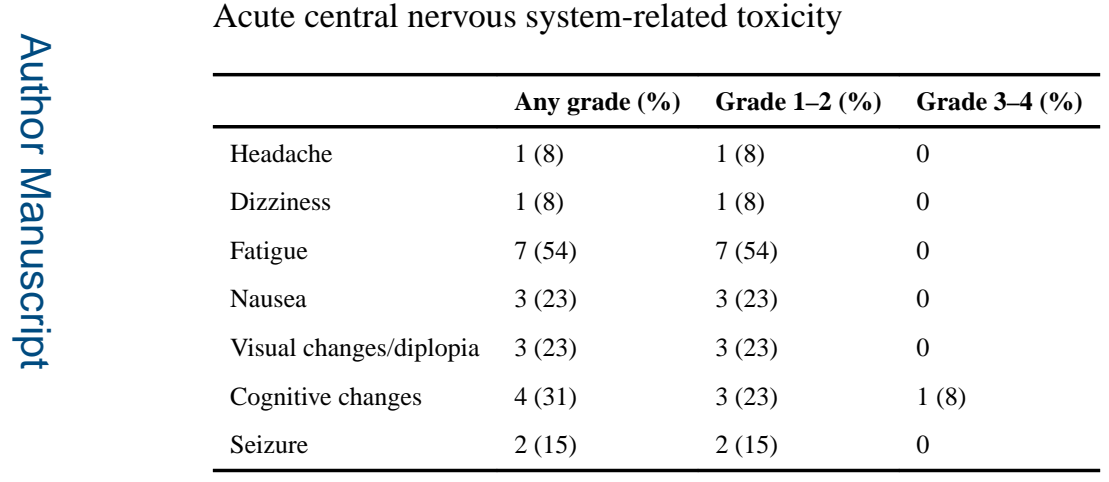

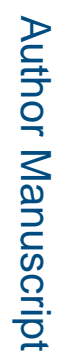

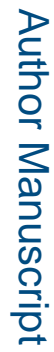

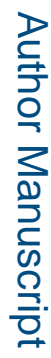

J Neurooncol. Author manuscript; available in PMC 2016 July 21. 